\title{
FROM THYROIDECTOMY TO THYROID CANCER: EPIDEMIOLOGICAL DATA FROM 2004 TO 2014.
}

An. Anyfantakis, E. Vourliotaki, E.M. Foukaki, S.Alexaki, M. Avloniti, K. Chatziriga, K. Stamataki.

Department of Endocrinology and Metabolism, Venizeleio General Hospital, Heraklio, Crete, Greece

\section{Introduction and Objectives}

Thyroid cancer has been rising all over the world in the last decades. This may reflect a true increase in the occurrence of disease, but may also be attributed to heightened medical surveillance and the use of improved diagnostics.

We evaluated the evolution of thyroid cancer epidemiological characteristics in our hospital from 2004 to 2014.

\section{Materials and Methods}

Retrospective analysis of histological data from thyroidectomies was performed in our hospital in the period 2004-2014.

SPSS was used for the statistical analysis.

\section{Results}

From 2004 to 2014, 2725 thyroidectomies (women 81\% versus men 19\%) were performed in our hospital.

In 1083 of these (40\%) histologic examination revealed thyroid cancer.

$34 \%$ of all cancers were diagnosed from 2004 to 2010 , while this percentage rises to $66 \%$ during the last four years (Fig. 1).

Median age at diagnosis was 49,2 years.

Histologic types: papillary $94 \%$, follicular 2,3\%, medullary 2,8\%, Hurtle-cell 0,6\%, anaplastic 0,1\% (Fig.2).

In almost half $(43,7 \%)$ of thyroid cancer patients, Hashimoto's thyroiditis coexisted (Fig 3$)$.

$48 \%$ of cancers were multifocal, whereas in the remaining $52 \%$ there was found a single focus on histologic examination (Fig. 4 ).

In the multifocal cancers, median maximum focus was less than $1 \mathrm{~cm}$ in $68,7 \%$ and $>1 \mathrm{~cm}$ in $31,3 \%$ (Fig. 5 ).

Invasion of thyroid capsule was reported in $36,7 \%$ of all cancers during the whole decade.

It's worth noting that, during the last 4 years, microcarcinomas (foci $<1 \mathrm{~cm}$ ) showed more aggressive behaviour, as far as thyroid capsule invasion is concerned (Fig. 6).

$26,5 \%$ showed extrathyroidal expansion, independently of the maximum focus size (Fig. 7).

It's interesting to report that frequency of invasive behaviour is increasing from 2008-2014 (Fig. 8 )

\section{Figures}
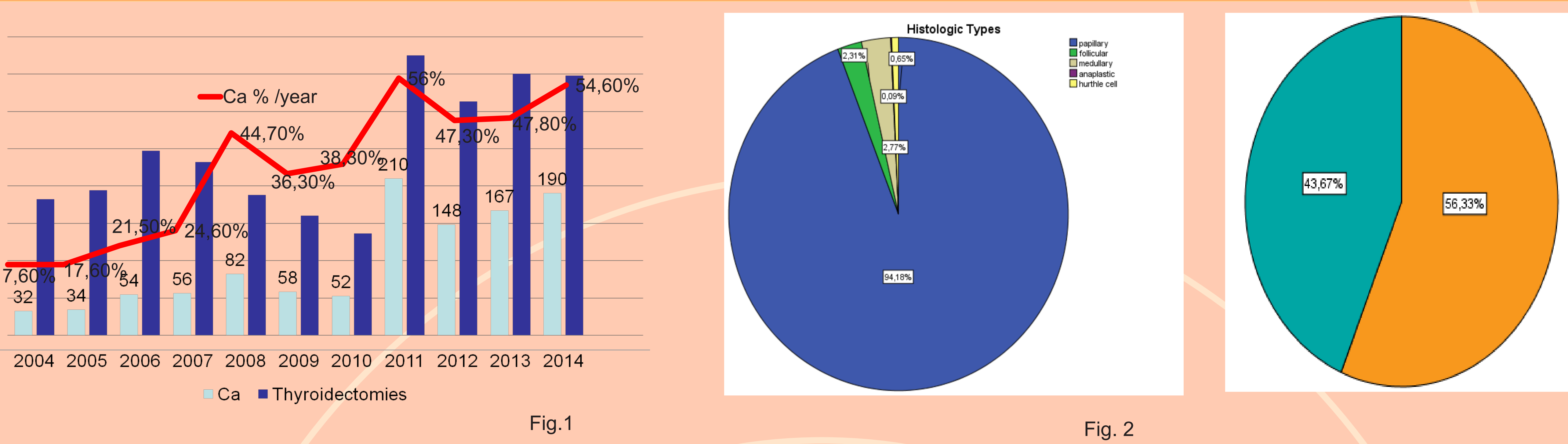

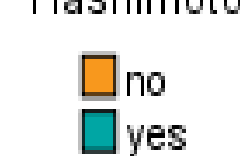

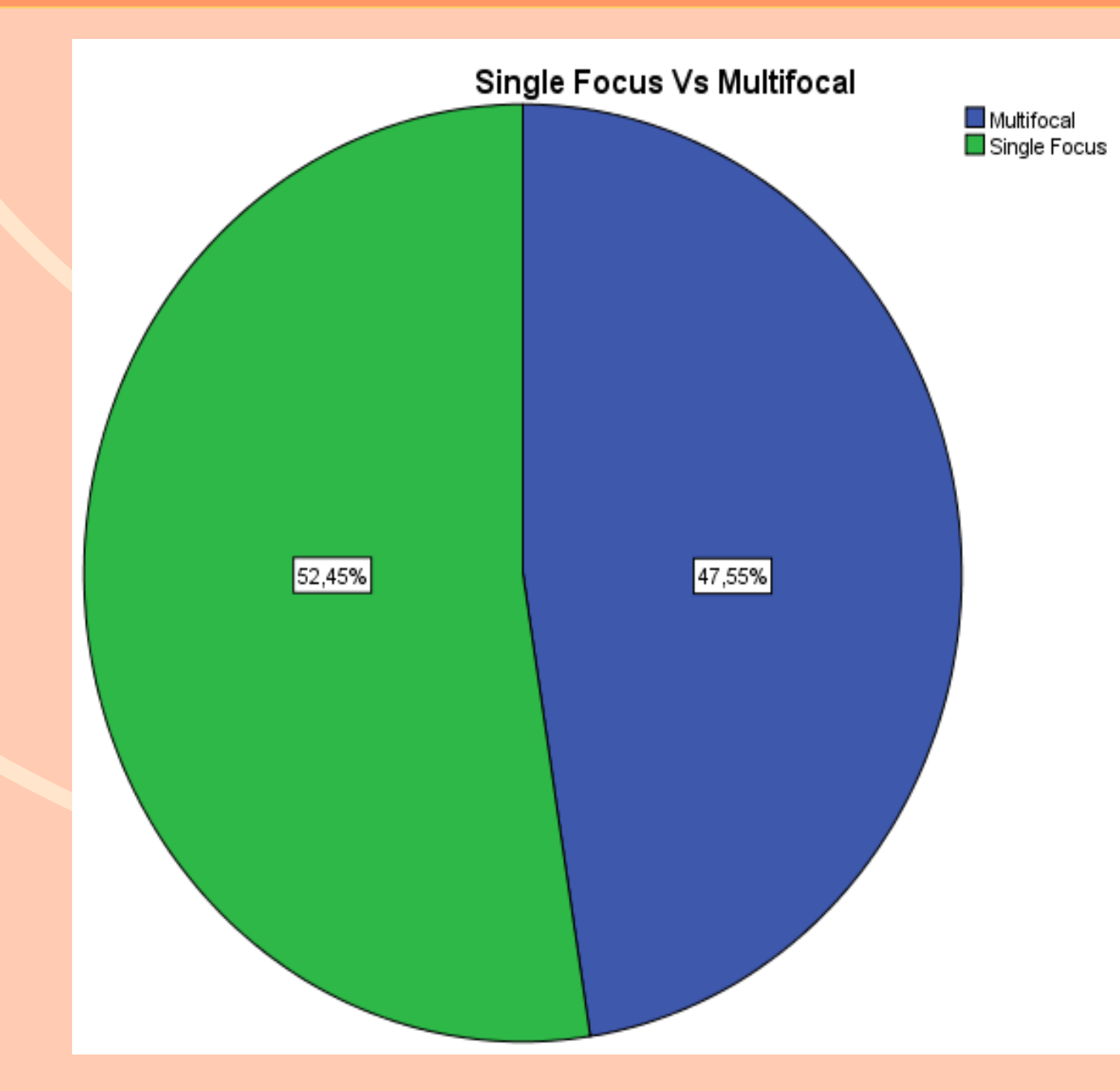

Fig. 2

Fig. 3

Fig. 4

Fig.5
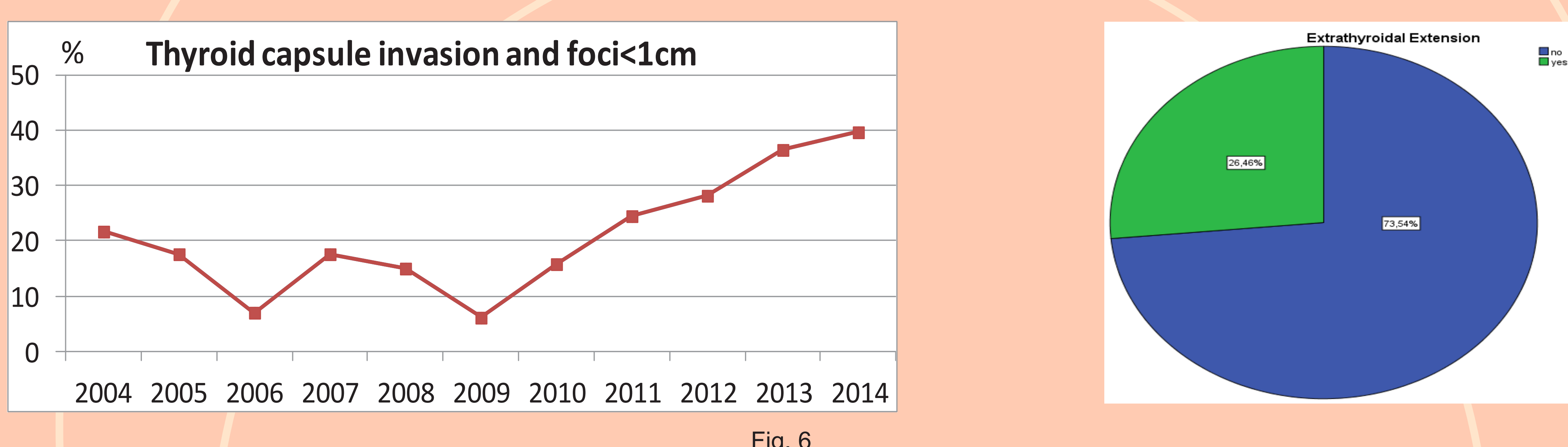

\section{Conclusions}

- The percentage of thyroid cancers is gradually increasing per year in the period studied, which could mean:

- more targeted pre-surgery diagnostic evaluation and referral for thyroidectomy.

- a possible real increase in thyroid cancer frequency.

- Maximum ferquency of thyroid cancer is reported in middle aged patients, mainly women.

- Hashimoto's thyroiditis coexists in almost $50 \%$ of cases.

- Independently of maximum thyroid cancer focus, increased frequency of invasive behaviour is reported during the last 5 years.

$\begin{array}{lll} & \text { 1. } & \text { Pellegriti G, J Cancer Epidemiol. 2013; Article ID } 965212\end{array}$

References:
Enewold L, Cancer Epidemiol Biomarkers Prev. 2009;18:784

Sipos JA, Clin Oncol (R Coll Radiol). 2010;22:395
5. Morris LG, Thyroid. 2013;23:885

6. Siegel R, 2014. CA Cancer J Clin. 64:9

7. Davies L, 2014. JAMA Otolaryngol Head Neck Surg. 140:317

8. Leenhardt L, 2004. Eur J Endocrinol. 150:133 